\title{
Clinical Profile and Management of Peritonsillar Abscess in a Sub Saharan Africa
}

\author{
Waheed Atilade Adegbiji ${ }^{1}$, Gabriel Toye Olajide ${ }^{* 2}$, Aluko AbdulAkeem Adebayo ${ }^{3}$ \\ ${ }^{1}$ ENT Department, Ekiti State University Teaching Hospital, Ado Ekiti ,Nigeria; awagbiji@ yahoo.com \\ ${ }^{2}$ ENT Department, Federal Teaching Hospital Ido-Ekiti, Nigeria/Afe-Babalola University College of Medicine and Health \\ Sciences, Ado-Ekiti/Nigeria; toyeolajide@ yahoo.co.uk \\ ${ }^{3}$ ENT Department, Aminu Kano Teaching Hospital, Kano, Nigeria; abdulakeemaluko@ gmail.com
}

Corresponding Author: Dr Gabriel Toye Olajide; toyeolajide@ yahoo.co.uk

Received 08 December 2019;

Accepted 27 December 2019;

Published 09 January 2020

\begin{abstract}
Background: Peritonsillar abscess is a common pathology that presents to an otolaryngologist with poor documentation especially in developing countries.

The aim of this study was to describe the clinical profile and management of peritonsillar abscess in our practice.

Material and Method: This was a retrospective study of patients presenting to our Ear, Nose and Throat facility with diagnosis of peritonsillar abscess. Information obtained includes detailed demographic characteristics, clinical findings and management of the patients. All obtained data were collated, entered into computer system and analyzed using SPSS version 18.0.

Results: Prevalence of peritonsillar abscess was $0.2 \%$. There were $38.9 \%$ males and male to female ratio was $1: 1.6$. Duration of illness at presentation with more than 1 week was $75.9 \%$ and single episode occurred in $85.2 \%$. Right peritonsillar abscess accounted for $66.7 \%$ and aspirated pus of more than $5 \mathrm{ml}$ was recorded in $48.1 \%$. Majority (87.0\%) of the patients was referred and $42.6 \%$ of such referral is by family physician. Patients seen in ear, nose and throat outpatient clinic were $83.3 \%$.

Common symptoms at presentation were fever, sore throat, odynophagia and otalgia in $100 \%, 94.4 \%, 90.7 \%$ and $59.3 \%$ respectively. All the patients $(100 \%)$ had enlarged tonsils and with deviated uvula on clinical examination followed by $98.1 \%$ exudate on the tonsils and $31.5 \%$ trismus. Test aspirate confirmed peritonsillar abscess in $68.5 \%$ and all revealed growth of bacteria. Common isolated bacteria were $35.2 \%$ Streptococcus species and $11.1 \%$ Staphyloccocus aureus.

Isolated Streptococcus species and Staphylococcus aureus had 100\% sensitivity to cefuroxime, gentamicin, azithromycin, ceftazidine, Amoxicillin and Streptomycin.

Prior prehospital treatment was received by $81.5 \%$ patients. Hospital treatments were surgical intervention and medical treatment in $90.7 \%$ and $100 \%$ respectively. Recurrences occurred in $3.7 \%$ during followed up.

Conclusion: Peritonsillar abscess are common disorder with female preponderance in this study. The prevalence recorded in this study was $0.2 \%$. It usually mimic other causes of fever hence eye index of suspicion is essential. Surgical treatment remains the effective mode of management in our study.
\end{abstract}

Keywords: Profile, Peritonsillar abscess, Bacteriology, Management, Nigeria

\section{Introduction}

Peritonsillar abscess are acute infection of peritonsillar space secondary to mainly acute tonsillitis. Peritonsillar abscess is associated with collections of purulent material in peritonsillar space behind the tonsil and in front of superior constrictor and palatopharyngeus muscle. ${ }^{[1,2]}$ Usually there is a transition phase of the peritonsillar inflammatory process becoming an abscess or quinsy. No suppuration or collection in per tonsillitis. Both are however the most common complication of poorly or untreated

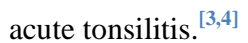

It is one of the most commonly occurring acute suppurative infections of the head and neck potential space in children and young adults worldwide. Peak incidence occurred in children in the second decade while in adults 20 to 40 years of age. ${ }^{[5,6]}$

This always first presented to the family physician and as emergency in the casualty department of the hospital. Appropriate skills and experience is required by untrained hand for diagnosis. Classical presentations are sore throat, odynophagia, neck pain, fever, malaise, otalgia and dysphagia. There is associated characteristic trismus accompanied with hot potato speech. ${ }^{[9,10]}$ Clinical examination of the oropharynx reveal marked asymmetry 
of the both tonsillar enlargement with erythema and oedematous peritonsillar area with displacement of the uvula to the contralateral side. ${ }^{[7]}$

Prompt recognition and initiation of therapy is important to avoid potential serious complications. Peritonsillar abscess is one of the head and neck infections in adults. The onset of peritonsillar abscess usually starts from superficial infections and progressively developing into tonsillar cellulitis. Peritonsillar abscess complications that may occur include expansion into parapharyngeal infection, mediastinitis, dehydration, pneumonia, intracranial infections such as cavernous sinus thrombosis, meningitis, brain abscess and airway obstruction. ${ }^{[8]}$

Peritonsillitis and subsequently peritonsillar abscess arises from complicated untreated or poorly treated acute tonsillitis. If they were mismanaged it leads to further untoward effects. An untreated or improperly treated case may leads to parapharyngeal space abscess, retropharyngeal abscess, sepsis, airway obstruction or death in severe cases. ${ }^{[11]}$

There is no data on patients with peritonsillar abscesses in south western Nigeria and has not been vastly discussed in the literature compare to developed countries. ${ }^{[12,13]}$ The aim of this study was to describe the clinical profile and management of peritonsillar abscess in south western Nigeria.

\section{Materials and Methods}

This was retrospective study carried out on all patients treated on account of peritonsillar abscess. The study was carried out by using the clinic and medical records of patients who came to Ear, Nose and Throat Department of Ekiti state university teaching hospital, Ado Ekiti, Nigeria. All the patients with peritonsillar abscess over a period of ten years, between January 2009 and December 2018 were studied. The teaching hospital apart from rendering a primary and secondary health care services, also serves as a referral center to surrounding states of Ondo, Kwara, Osun and Kogi. Records of all the patients (54) diagnosed as having peritonsillar abscess that were seen and treated in the Accident and Emergency unit, ENT clinic and wards was retrieved from the hospital medical record department. Information retrieved include their socio-demographic characteristics, presenting complaints, pre hospital treatment, past medical and surgical history were recorded. Detailed clinical findings on general examination, oropharyngeal, other otorhinolarygologic, head and neck were extracted and documented. Results of the test aspirate that was sent for microscopic, culture and sensitivity were also retrieved from the patient's case notes and recorded. Patients with incomplete medical records were excluded from this study. All obtained data were collated, entered into computer system and analyzed by SPSS version 18.0. The data were further tabulated and presented descriptively in frequency tables and charts. Permission for this study was obtained from the hospital Ethical and research committee.

\section{Results}

A total number of 17,681 patients were seen in ear, nose and throat department over the studied period out of which 54 patients had a diagnosis of peritonsillar abscess. Based on aspiration test, 37 $(68.5 \%)$ were confirmed to have peritonsillar abscess while 17 $(31.5 \%)$ showed evidence of peritonsillitis, given a prevalence of $0.2 \%$.
The age group 21 - 30 years has the highest number of patients (46.3\%) and the least (1.9\%) was recorded at age group of $\geq 41$ years. Figure 1

There were $21(38.9 \%)$ males and $33(61.1 \%)$ females. Male to female ratio was $1: 1.6$. Urban dwellers in $29(53.7 \%)$ were commoner than rural dwellers in $25(46.3 \%)$. Those that practice Christianity accounted for $48(88.9 \%)$ while Muslim faith accounted for $6(11.1 \%)$. Educational levels of the patients were 18 $(33.3 \%)$ post-secondary, $17(31.5 \%)$ primary and $13(24.1 \%)$ secondary. Patient's occupations were mainly 15 (27.8\%) business, $14(25.9 \%)$ artisan and $11(20.4 \%)$ students/apprentice. As demonstrated in table 1.

Large percentage of patient, 41 (75.9\%) presented after one week while $13(24.1 \%)$ presented in one week or less. Single episode of peritonsillar abscess occurred in 46 (85.2\%) while recurrence occurred in $8(14.8 \%)$. Right peritonsillar abscess accounted for $36(66.7 \%)$ while left peritonsillar abscess accounted for $18(33.3 \%)$. Aspirated pus of less than $5 \mathrm{ml}$ was recorded in 11 $(20.4 \%)$ while more than $5 \mathrm{ml}$ was recorded in $26(48.1 \%)$. As showed in table 2.

Majority $47(87.0 \%)$ of the patients were referred while selfreporting was $7(13.0 \%)$. Major sources of referral was by family physician in $23(42.6 \%)$ of patients. This was followed by 14 $(25.9 \%)$ paediatrician and $10(18.5 \%)$ casualty officer. As illustrated in figure 2 .

Emergency presentation was less common in this study. There were $45(83.3 \%)$ patients seen in ear, nose and throat outpatient clinic while $9(16.7 \%)$ were seen at the emergency ward. Common symptoms at presentation were fever, sore throat, odynophagia and otalgia in 54 (100\%), 51 (94.4\%), 49 (90.7\%) and $32(59.3 \%)$ respectively. Frequent signs on clinical examination were $54(100 \%)$ enlarged tonsils, $54(100 \%)$ deviated uvula, 53 $(98.1 \%)$ exudate on the tonsils and $17(31.5 \%)$ trismus. As shown in table 3 .

Aspirate was sent for microscopy, culture and sensitivity in those that have peritonsillar abscess. No bacteria were isolated from $8(14.8 \%)$ aspirate. Common isolated bacteria were 19 (35.2\%) Streptococcus species, 6 (11.1\%) Staphyloccocus aureus, $3(5.6 \%)$ Klebsiella species and H. Influenza 1(1.8\%). This is demonstrated in figure 3 .

Isolated Streptococcus species and Staphylococcus aureus from the aspirates were $100 \%$ sensitivity to cefuroxime, gentamicin, azithromycin, ceftazidine, Amoxicillin and Streptomycin while Staphylococcus aureus further had 100\% sensitivity to Ampicillin and Cephalexin. Both showed some sensitivity to Floxapen and co-trimoxazole. As illustrated in table 4.

Prior treatment was received by $44(81.5 \%)$ patients and these treatments were received through herbal in $22(40.7 \%)$ patients, $19(35.2 \%)$ over the counter and $13(24.1 \%)$ pharmacy. Hospital treatments were surgical intervention and medical treatment in $49(90.7 \%)$ and $54(100 \%)$ respectively. Surgical treatments were $49(90.7 \%)$ needle aspiration, $32(59.3 \%)$ incision and drainage and $13(24.1 \%)$ tonsillectomy. Medical treatment were antibiotics, analgesic and gargle in all patients. Recurrence occurred in $2(3.7 \%)$ during followed up. The recurrences occurred in multiple episode patients and were noticed after 3 months of follow up. 
Figure 1: Showing age group (years)

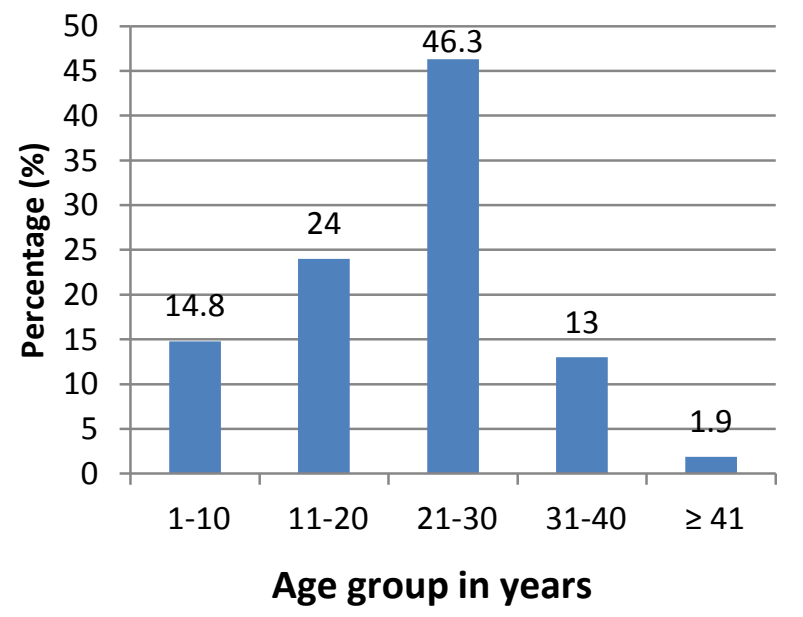

Table 1: Sociodemographic features of the patients

\begin{tabular}{|l|c|c|}
\hline Sociodemographic features & Number & Percentage (\%) \\
\hline Sex & 21 & 38.9 \\
Male & 33 & 61.1 \\
Female & & \\
Dwelling & 29 & 53.7 \\
Urban & 25 & 46.3 \\
Rural & & \\
Religion & 48 & 88.9 \\
Christian & 6 & 11.1 \\
Muslim & & \\
Education level & 6 & 11.1 \\
Nil formal & 17 & 31.5 \\
Primary & 13 & 24.1 \\
Secondary & 18 & 33.3 \\
Post-secondary & & \\
Parent/patient Occupation & 11 & 20.4 \\
Students/apprentice & 15 & 27.8 \\
Business & 14 & 25.9 \\
Artisan & 9 & 16.7 \\
Civil servant & 6 & 11.1 \\
Farming & & \\
\hline
\end{tabular}

Table 2: Characteristics of peritonsillar abscess among patients

\begin{tabular}{|l|c|c|}
\hline Characteristics & Number & Percentage (\%) \\
\hline Duration prior to & & \\
presentation & 13 & 24.1 \\
$<1$ week & 41 & 75.9 \\
$>$ 1week & 46 & 85.2 \\
Recurrency & 8 & 14.8 \\
1 episode & & \\
Recurrent & 36 & 66.7 \\
Laterality & 18 & 33.3 \\
Right & & \\
Left & 17 & 31.5 \\
Volume of pus & 11 & 20.4 \\
Nil & 26 & 48.1 \\
$<5 \mathrm{ml}$ & & \\
$>5 \mathrm{ml}$ & & \\
\hline
\end{tabular}

\section{Figure 2 : Sources of referral among patients}

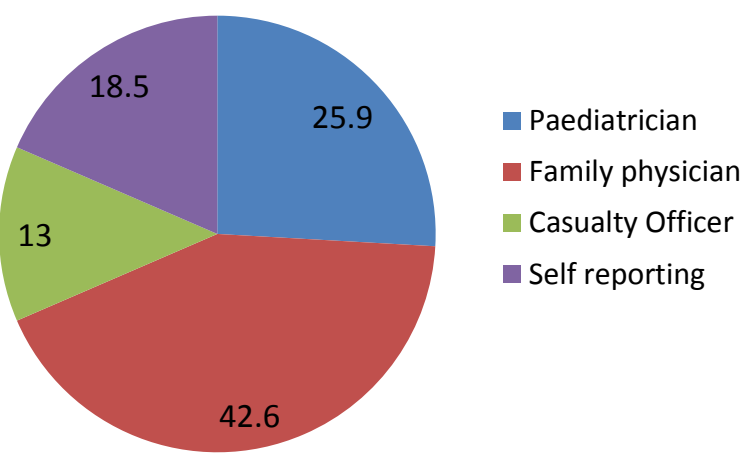

Table 3: Clinical features among patients

\begin{tabular}{|l|c|c|}
\hline Clinical features & Number & $\begin{array}{c}\text { Percentage } \\
(\%)\end{array}$ \\
\hline Sore throat & 51 & 94.4 \\
Otalgia & 32 & 59.3 \\
Neck pain/cervical lymphadenopathy & 16 & 29.6 \\
Trismus & 17 & 31.5 \\
Odynophagia & 49 & 90.7 \\
Enlarged tonsils & 54 & 100 \\
Deviated uvula & 54 & 100 \\
Fever & 54 & 100 \\
Exudate & 53 & 98.1 \\
\hline
\end{tabular}

NB: Some patient presented with more than one clinical features

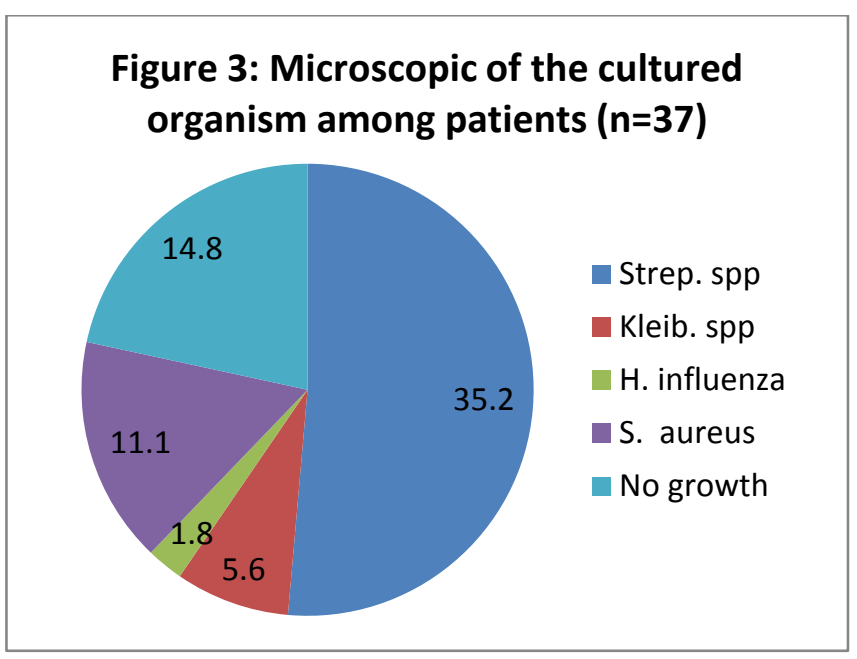

Table 4: Antibiotic sensitivity among the patients

\begin{tabular}{|l|c|c|c|c|}
\hline Sensitivity & $\begin{array}{c}\text { Strept. } \\
\text { species } \\
\mathrm{n}(\%)\end{array}$ & $\begin{array}{c}\text { Staphyl. } \\
\text { aureus } \\
\mathrm{n}(\%)\end{array}$ & $\begin{array}{c}\text { Klebsiella } \\
\text { species } \\
\mathrm{n}(\%)\end{array}$ & $\begin{array}{c}\text { Haemop } \\
\text { hilus } \\
\text { influenza } \\
\mathrm{n}(\%)\end{array}$ \\
\hline Cefuroxime & $19(100)$ & $6(100)$ & $3(100)$ & $1(100)$ \\
Gentamicin & $19(100)$ & $6(100)$ & $2(66.7)$ & $1(100)$ \\
\hline Azithromycin & $19(100)$ & $6(100)$ & $3(100)$ & $1(100)$ \\
Ceftazidine & $19(100)$ & $6(100)$ & $3(75.0)$ & $1(100)$ \\
\hline Amoxicillin & $19(100)$ & $6(100)$ & $2(66.7)$ & $1(100)$ \\
Streptomycin & $19(100)$ & $6(100)$ & $2(66.7)$ & $0(0)$ \\
\hline Ampicillin & $14(73.7)$ & $6(100)$ & $2(66.7)$ & $0(0)$ \\
Cephalexin1 & $14(73.7)$ & $6(100)$ & $2(66.7)$ & $0(0)$ \\
\hline Floxapen & $12(63.2)$ & $3(50.0)$ & $1(33.3)$ & $0(0)$ \\
Cotrimoxazole & $2(10.5)$ & $2(33.3)$ & $0(0)$ & $0(0)$ \\
\hline
\end{tabular}




\section{Discussion}

Peritonsillar abscess is a common clinical condition, and usually secondary to complicated tonsillitis with low prevalence of $0.2 \%$ in this study. This observed prevalence may be as a result of lower reported cases in the hospital. Many cases seek herbal intervention; traditional surgical treatment and self-medication. ${ }^{[14]}$ when such non-orthodox measures failed and perhaps complication sets in, such patients seek specialist care.

The age distribution of patients was peaked at third decade and lowest age group distribution was above 40 years. Peritonsillar abscess are common in adults and it is rare in children, except in children who are immunocompromised. Similar report was reported in previous study. ${ }^{[15]}$ Younger adult age group was observed in other study. ${ }^{[16]}$ There was female preponderance over male in our findings. Propably female at this age group are more prone to acquired upper respiratory infection from their children. Our study is contrary to other study with male preponderance. ${ }^{[17]}$ Other study revealed equal distribution of male and female sexes. ${ }^{[18]}$ Majority of the studied patients were urban dwellers this may be due to easy accessibility of our center in the state capital.

Majority of our patients reported more than a week after onset of their symptoms. The patients were wrongly diagnosed as cases of plasmodiasis (malaria) or enteric fever. They were poorly treated until the sore throat progresses to severe odynophagia and trismus as noted in this study. First episode of peritonsillar abscess were commoner than recurrent cases. Poorly and inadequate drainage with defective anatomy from poorly healed scar serve as loci for reinfection. Cases of both first episode and recurrent cases were reported in previous studies. ${ }^{[19,20]}$ All our patients presented with unilateral peritonsillar abscess and right side was predominantly affected. This finding is contrary to other retrospective study with predominant left peritonsillar abscess. ${ }^{[21]}$ The incidence of bilateral peritonsillar abscess was reported in other study. ${ }^{[22]}$ Aspirated pus was more than $5 \mathrm{ml}$ in majority of the patient which may be due to predominantly adults and late presentation. In most of these patients fine needle aspiration of the pus without incision and drainage was adequate for their treatment.

Most of the peritonsillar abscess patients were mainly referred by the first contact physician as compared to few cases that were self-reporting. These indicate the significance of family physician, paediatrician and emergency doctors in diagnosis and treatment of this disorder at early stage.

The entire patients were on analgesics with associated pain relief prior to presentation. Most of the patients also volunteer that it is only the analgesics that enable them to feed and sleep. This may be the reasons why most of our patients presented very late to ear, nose and throat outpatient clinic. Few patients presented as emergency in the casualty department to casualty officer.

Patients with peritonsillar abscess mainly presented with fever, sore throat, enlarged tonsils, deviated uvula, odynophagia, exudate on tonsils, otalgia and trismus as demonstrated by other previous studies. ${ }^{[17,18,23]}$ These symptoms ranges from those of simple infection to those of severe and complicated infection of the tonsils. Fever and sore throat occurred in acute tonsillitis while odynophagia with trismus occurred in peritonsillar abscess due to inflammation and spasm of muscles of mastication.

In this study, test aspirate was done to confirm peritonsillar abscess and to rule out other differential. Based on microbiological culture of the peritonsillar aspirate, Streptococcus species and Staphylococcus aureus were the main organisms isolated. This results is similar to findings from other studies. ${ }^{[24,25]}$ Streptococcus species and Staphylococcus aureus that were obtained are most sensitive to the antibiotic like Cefuroxime, Gentamicin, Azithromycin, Ceftazidine, Amoxicillin and Streptomycin. There are variable resistance to Ampicillin, Cephalexin, Floxapen and Cotrimoxazole. This is supported by findings from study in Nigeria. ${ }^{[26]}$

Majority of these patients had wrong prehospital treatment by using antimalaria and analgesics. Most of the drugs were from herbalist, over the counter and pharmacy with poor outcome. Our surgical interventions were needle aspiration to confirm the diagnosis, definitive treatment and obtain aspirate for microscopy, culture and sensitivity. Incision and drainage were performed for majority with inadequate fine needle drainage of pus while tonsillectomy was carried out for patients with recurrent peritonsillar abscess or associated recurrent tonsillitis. All our patients with peritonsillar abscess were offered medical treatment. Antibiotic inform of clavulinated amoxicillin parenteral or oral administered depending on the severity of the odynophagia. Parenteral or oral analgesic was given depending on the degree of the pain. All patients had diluted hydrogen peroxide or warm saline gargle routinely. All patients were followed up in ear, nose and throat outpatient clinic. During follow up two patients had recurrent episode and were offered tonsillectomy after treatment of acute infection.

\section{Conclusion}

Peritonsillar abscess are common disorder with female preponderance in this study. The prevalence recorded in this study was $0.2 \%$. It usually mimic other causes of fever hence eye index of suspicion is essential. Surgical treatment remains the effective mode of management in our study.

\section{Funding}

There was no financial support. It is a self-sponsored research study.

\section{Competing interests}

All the authors declare that there were no competing interests.

\section{Acknowledgements}

The authors are most grateful to Ekiti state university teaching hospital, the staff and all the patients who participated in this study.

\section{Reference}

[1] Papacharalampous GX, Vlastarakos PV, Kotsis G, Davilis D, Manolopoulos L. Bilateral peritonsillar abscess: A case presentation and review of the current literature with regard to the controversies in diagnosis and treatment. Case Reports in Medicine. 2011;1-4.

[2] Segal N, Sabri SE. Peritonsillar abscess in children in the southern district of Israel. Int Journal of Ped Otol. 2009; 73:1148-50.

[3] Salihoglu M, Eroglu M, Yildirim AO, Cakmak A, Hardal U, Kara K. Transoral ultrasonography in the diagnosis and treatment of peritonsillar abscess. Clinical Imaging. 2013; 37:465-7.

[4] Marom T, Cinamon U. Changing trends of peritonsillar abscess. Am J of Otol HNS. 2010; 31:162-7. 
[5] Khayr W, Taepke J. Management of peritonsillar abscess: needle aspiration versus incision and drainage versus tonsillectomy. Am J Ther. 2005; 12(4):344-350.

[6] Steyer TE. Peritonsillar abscess: diagnosis and treatment. Am Fam Physician. 2002; 65(1):93-96.

[7] Farmer SEJ, Khatwa MA, Zeitoun HMM. Peritonsillar abscess after tonsillectomy: a review of the literature. Ann R Coll Surg Engl. 2011; 93(5):353-357.

[8] Naik SM, Naik SS. Interval tonsillectomy: 27 Cases of peritonsilar abscess managed in Medical College Hospital. Indian Journal of Clinical Practice. 2013; 24(4):342-6.

[9] Kara N, Spinou C. Appropriate antibiotics for peritonsillar abscess: A 9 months cohort. Otorhinolaryngologia-Head and Neck Surgery. 2010; 40:20-4.

[10] Wang YP, Wang MC, Lin HC, Chou P. The impact of prior tonsillitis and treatment modality on the recurrence of peritonsillar abscess: A nationwide cohort study. Recurrence of Peritonsillar Abscess in a Nationwide Cohort Study. 2014; 9:1-7.

[11] Bartlett A, Bola S, Williams R. Acute tonsillitis and its complications: an overview. J R Nav Med Serv. 2015; 101(1):69-73.

[12] Powell J, Wilson JA. An evidence-based review of peritonsillar abscess. Clin Otolaryngol. 2012; 37(2):136145 .

[13] Megalamani SB, Suria G, Manickam U, Balasubramanian D, Jothimahalingam S. Changing trends bacteriology of peritonsillar abscess. J Laryngol Otol. 2008; 122(9):928-930.

[14] Olajide GT, Olajuyin OA, Adegbiji WA. Traditional Uvulectomy: Origin, Perception, Burden and Strategies of Prevention. International Journal of Medical Reviews and Case Reports (ARTICLE IN PRESS).

[15] Ozbek C, Aygenc E, Tuna EU, Selcuk A, Ozdem C. Use of steroids in the treatment of peritonsillar abscess. The Journal of Laryngology \& Otology. 2004; 118:439-42.

[16] Love RL, Allison R, Chambers ST. Peritonsillar infection in Christchurch 2006-2008: epidemiology and microbiology. The New Zealand Medical Journal. 2011; 124:1336.

[17] Iqbal SM, Husain A, Mughal S, Khan IZ, Khan IA. Peritonsillar cellulites and quinsy, clinical presentation and management. Armed Forces Med J. 2009;59(4):27580 .

[18] Khan MI, Khan A, Muhammad. PERITONSILLAR ABSCESS: CLINICAL PRESENTATION AND EFFICACY OF INCISION AND DRAINAGE UNDER LOCAL ANAESTHESIA. J Ayub Med Coll Abbottabad. 2011; 23(4):34-6.

[19] Seyhun N, Batur Çalış ZA, Ekici M, Turgut S. Epidemiology and Clinical Features of Peritonsillar Abscess: Is It Associated with Seasonal Variations? Turk Arch Otorhinolaryngol 2018;10.5152/tao.2018.3362.

[20] Powell J, Wilson JA. An evidence-based review of peritonsillar abscess. Clin Otolaryngol. 2012; 37:136-45.

[21] Tyagi V, Kaushal A, Garg D, De S, Nagpure P. Treatment of peritonsillar abscess- A prospective study of aspiration versus incision and drainage. Calicut Med J. 2011; 9(3):e3.

[22] Watanabe T, Suzuki M. Bilateral peritonsillar abscesses: our experience and clinical features. Ann Otol Rhinol Laryngol. 2010; 10:662-6.

[23] Rahayu L, Putra A. E, Sari S. The Distribution of Peritonsillar Abscess Patients in Sanglah Hospital Denpasar at 2010 until 2017. Biomed Pharmacol J. 2017; 10(2).

[24] Repanos C, Mukherjee P, Alwahab Y. Role of microbiological studies in management of peritonsillar abscess. The Journal of Laryngology \& Otology. 2009; 123:877-9.

[25] Ryan S, Papanikolaou V, Keogh I. Appraisal of the perihospital management and evolving microbiology of peritonsillar abscess disease. B-ENT. 2014; 10(1):15-20.

[26] Okoye EL, Obiweluozor CJ, Uba BO, Odunukwe FN. Epidemiological Survey of Tonsillitis Caused By Streptococcus pyogenes among Children in Awka Metropolis (A Case Study of Hospitals in Awka Community, Anambra State). IOSR Journal of Pharmacy and Biological Sciences. 2016; 11(3):54-58. 